\title{
Diaphragm postural function in injured subtalar joint
}

\author{
doi: https://doi.org/10.5114/pq.2018.73369
}

\author{
Marcin Urbaniak, Aleksandra Kisilewicz, Sebastian Klich \\ Department of Paralympic Sports, Faculty of Sports Science, University School of Physical Education in Wrocław, \\ Wrocław, Poland
}

\section{Abstract}

Introduction. Loss of stability and persistent swelling in patients with subtalar joint injury occur very often. Diaphragm plays an important role in providing proprioceptive information and its proper function remains a key factor reducing swelling in the distal part of the body. The aim of this study was to evaluate the influence of diaphragm osteopathic manipulative treatment (OMT) on postural control and swelling reduction.

Methods. The study is a case report describing a 23-year-old male who injured his right subtalar joint during a football match. Before the therapy, a complex physical examination was performed: muscle strength and range of motion of lower limbs evaluation, initial testing of postural control, and the level of swelling determination with the figure-of-eight method. After extensive diagnosis, two OMT techniques were used: doming the diaphragm technique and thoracic pump with respiratory assist technique. Subsequently, the second static stability test and figure-of-eight method were applied.

Results. The length of the centre of pressure (COP) path with eyes opened decreased by $15 \%$, and with eyes closed by $45 \%$. The surface area circled by the COP path with eyes open decreased by $22 \%$, and with eyes closed by $68 \%$. There was no immediate change in swelling.

Conclusions. The study confirmed the efficacy of diaphragm OMT in improving postural control. Screening for diaphragm dysfunction should be part of complete osteopathic assessment in subtalar joint injury.

Key words: OMT, diaphragm, case report, osteopathy, postural stability

\section{Introduction}

Every level of sport performance requires appropriate training. Lack of warm-up, no previous mental and physical preparation may lead to physical injury [1]. It is very important to collect full medical history of the patient, including type of work and sports activity. Those are the keys to accurate diagnosis. It appears that elite football players use proprioceptive skills more efficiently and this may be considered as a performance criterion [2]. Typical football injuries in males refer to ankles (20\%), upper leg (17\%), and knee (15\%) [3].

Manual manipulation of the diaphragm is a technique used by both manual therapist and osteopaths. It has been reported effective in patients with pulmonary disease, as well as those with short hamstring syndrome [4-6]. On the basis of an anatomical concept, the use of osteopathic diaphragm manipulation and thoracic pump technique in swollen ankle and postural imbalance may be reasoned [7]. It is also supported by the fact that aortic hiatus is at the T12 level and contains the aorta, thoracic duct, azygos and hemiazygos veins [8]. Lymphatic vessels rely primarily on extrinsic mechanisms, such as the skeletal muscle pump, the respiratory pump and the pulse of nearby blood vessels, to facilitate lymph return [9]. Lymphatic absorption depends in the first place on the rhythmicity and stretching of the diaphragm [10] as lymph must be actively pumped against the pressure gradient [11]. Therefore, abdominal diaphragm contraction may produce changes in abdominal and thoracic cavities affecting pressure gradients and lymphatic flow [12, 13]. Lymph re-enters the cardiovascular system by way of the central thoracic duct that drains into subclavian veins [9, 14]. Summing up, as the whole lymph from the lower part of the body enters the thoracic duct through abdomen lymphatic vessels, diaphragm proper functioning remains a key factor reducing swelling in the distal part of the body.

Diaphragm is also responsible for trunk stabilization by its connection to the lumbar spine [10]. The medial arcuate ligaments extend over the anterior psoas muscles as fibrous attachments between the L1 or L2 vertebral body and the transverse processes of L1 [8]. It is part of the system that creates a hydraulic effect in the abdominal cavity, which assists spinal stabilization by stiffening the lumbar spine through increased intra-abdominal pressure [15]. Moreover, the stability of spine is established before the execution of a postural task by the central mechanism of anticipatory postural adjustments [16]. It occurs independently from the respiratory activity of the diaphragm [17].

In this article, both postural and lymphatic role of the diaphragm will be investigated in a 23-year-old amateur football player whose lymphatic and postural balance systems were damaged as a result of a subtalar joint injury. The aim of the study was to evaluate if the diaphragm might influence the postural control in the injured subject.

\section{Subjects and methods}

A 23-year-old man, whose details are kept anonymous, presented to a physical therapist on the recommendation of his medical doctor after experiencing pain and swelling

Correspondence address: Marcin Urbaniak, Department of Paralympic Sports, Faculty of Sports Science, University School of Physical Education in Wrocław, ul. Witelona 25a, 51-617 Wrocław, Poland, e-mail: marcinurbaniak@hotmail.com 
in his right ankle. During a game, after jumping to the ball, he had fallen on his right extended extremity and felt acute pain; swelling appeared immediately. It was his first injury of the extremity, no other injuries or operations were reported. The patient admitted playing football after the injury with his extremity swollen. He used a swelling reducing gel. The pain did not affect the patient's routine activities of daily living (except rare squat - pressure feeling in the subtalar joint region). The patient did not report any dizziness, loss of consciousness or stability problems. His social, medical, and family history were non-contributory.

Physical examination revealed normal muscle strength $5 / 5$ in Lovett scale - of the lower limbs bilaterally. The passive range of motion of the lower limb was normal on the left side. The comparison of both lower limbs showed that there was dorsal flexion restriction of $60 \%$ in the right ankle. The right hip was externally rotated about $40 \%$ more than the left one. The range of motion was measured with a goniometer; the method by Jung and Yamasaki [18] was applied.

As a proposed therapeutic technique and as part of extensive diagnostics, osteopathic manipulative treatment (OMT) was used. It was addressed to identified somatic dysfunction, which is defined as impaired or altered function of related components of the somatic system: skeletal, arthrodial, and myofascial structures, and related vascular, lymphatic, and neural elements [19]. For somatic dysfunctions, there must be four criteria present: tissue texture abnormality, asymmetry, restriction of motion, and tenderness [20]. All of those criteria were present in the patient being described. The osteopathic structural examination identified the right fibula drop, calf muscles spasm, interosseous membrane tightness, left side of symphysis pubis elevation and posterior rotation.

Doming the diaphragm technique and the thoracic pump with respiratory assist technique were chosen to improve the function of the lymphatic system and postural control. The techniques were performed as presented below.

\section{Doming the diaphragm}

Before the therapy, thoracic cage motion was assessed bilaterally by palpating the rib cage while the patient inhaled and exhaled. During treatment, the patient lay supine on a table. He was instructed to take a deep breath and then breathe all the way out. As the patient exhaled, the therapist followed the diaphragm by pressing thumbs posterior towards the table. A further cephalad motion of the diaphragm was continued during the next exhalation. It was repeated for five respiratory cycles, until the diaphragm domed easily at the end of exhalation.

\section{Thoracic pump with respiratory assist}

As previously, the thoracic cage motion was assessed before starting the treatment, and the patient lay supine on a table. He was instructed to inhale and exhale deeply. The therapist provided compressive force downward onto the chest and then oscillated the degree of compression to produce a pump motion. It was continued for approximately one minute. Respiratory assist was initiated by instructing the patient to inhale deeply and then exhale deeply. During the exhalation phase, the therapist followed the chest wall down until exhalation was completed, and then provided resistance while the patient begun inhalation. It was repeated for five respiratory cycles. During the final inhalation phase, right before the patient completed a full deep inhalation, the therapist rapidly removed the hands from the patient's chest to allow for a sudden influx of air into the chest [4].

A static stability test was conducted on a stabilometric platform (ALFA PRO PLUS). A static two-leg standing test was performed before and after the OMT treatment: for $30 \mathrm{sec}-$ onds with both, eyes open and closed. During the test, the path and surface area of the centre of pressure (COP) were measured. To determine the level of ankle joint swelling, the figure-of-eight method was used before and after the OMT treatment. It was proven to be a reliable and valid indirect tool in the assessment of ankle swelling after sprains or other lower-extremity musculoskeletal disorders [21, 22].

\section{Ethical approval}

The research related to human use has been complied with all the relevant national regulations and institutional policies, has followed the tenets of the Declaration of Helsinki, and has been approved by the authors' institutional review board or an equivalent committee.

\section{Informed consent}

Informed consent has been obtained from the patient included in this study.

\section{Results}

Twenty minutes after the treatment, there was a significant difference in postural sway observed (Figures 1-4). The length of the COP path with eyes open decreased by $15 \%$, and the length of the COP path with eyes closed decreased by $45 \%$. The surface area circled by the COP path with eyes open decreased by $22 \%$, and the surface area circled by the COP path with eyes closed decreased by $68 \%$. There was no immediate change in swelling.

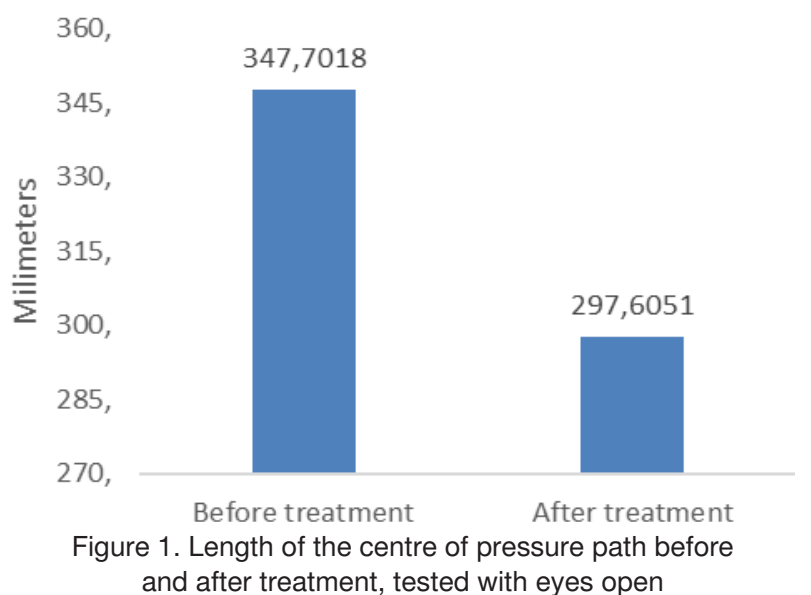

875,

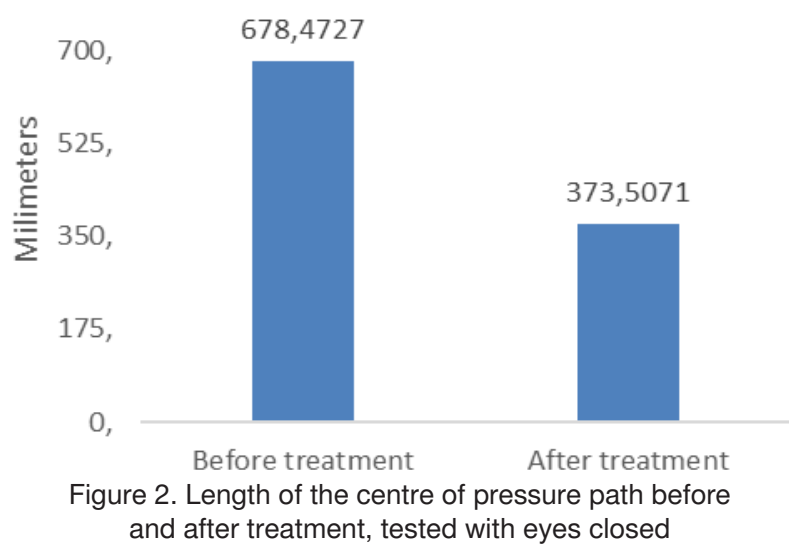




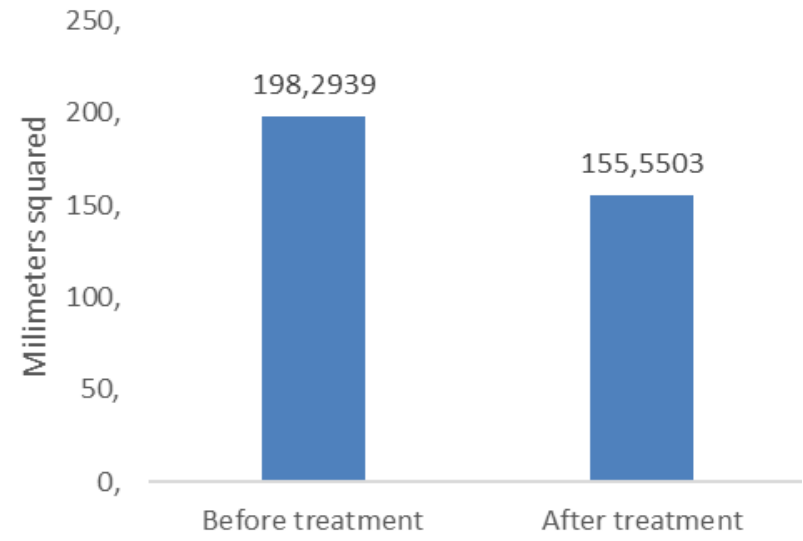

Figure 3. Surface area before and after treatment, tested with eyes open

875 ,

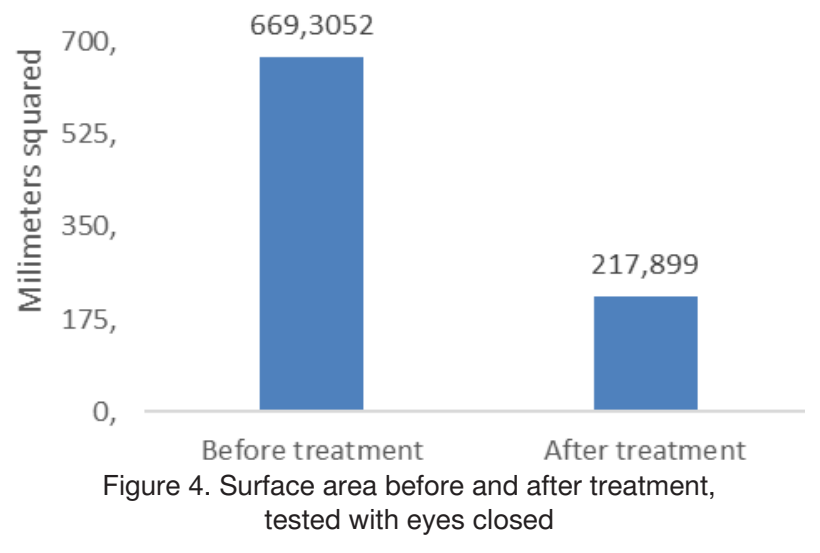

\section{Discussion}

Considering the main problem reported by the patient, the treatment was focused on the improvement of postural control and reduction of swelling. Given the fact that the diaphragm role is both lymphatic and postural, OMT was chosen as a proper therapeutic technique. Before the first treatment, the patient complained that he could not bear his weight equally on both lower extremities. After the treatment, the patient did not report this complaint and could stand on both feet equally. As the path and surface area of the COP decreased, the results indicate that there was an improvement in the subject's postural control. However, the goal of swelling reduction in the ankle was not achieved immediately.

Modified sensorimotor and postural control after ankle injuries has already been scientifically explained; it is caused by altered sensory inputs from the somatosensory system around the ankle [23]. Interestingly, insufficient ability to coordinate muscles surrounding the hip joint has also been observed after ankle joint injuries, with the influence on trunk and postural stability $[24,25]$. That would explain the external rotation of the hip in the presented patient. Terada et al. [26] examined 27 individuals with chronic ankle instability. They showed the association between chronic ankle instability and altered diaphragm contractility. Their study is therefore in agreement with our assumptions that the diaphragm muscle contributes to trunk and postural stability by modulating the intra-abdominal pressure. They also pointed the lack of previous research describing this particular connection.

Vostatek et al. [27] found a postural effect of diaphragm pared with 17 individuals suffering from low back pain, and MRI was applied to investigate the diaphragm activity. Kolar et al. [28] observed that altered postural activity of the diaphragm during the postural task of isometric resistance to the extremities might constitute a mechanism of chronic low back pain. This study was also performed with the use of MRI and respiratory measurements. What is more, specific loading of inspiratory muscles attenuated postural control by reducing lumbar proprioceptive sensitivity, forcing a proprioceptive use of the dominant ankle. Therefore, in this particular case, ankle proprioception was disturbed. It was proven that for all dynamic activities, from simple functional tasks to skilled athletic manoeuvers, proper stabilization is critical [17].

Hodges et al. [29] concluded that the postural activity of the diaphragm might be decreased when the respiratory demand was increased if the abdomen or thorax was stiffened by other activities. In their study, 13 volunteers were tested with electromyography of the diaphragm and other trunk muscles and respiratory measurement to determine how those parameters changed during arm movement. Janssens et al. [30] also showed that proprioceptive postural control might be improved by enhancing the trunk stabilizing function of the diaphragm with inspiratory muscle training (IMT). In their study, 28 individuals with low back pain were randomly assigned to 2 groups - high intensity IMT or low intensity IMT. Proprioception use was tested by measuring the COP displacement during local muscle vibration at the beginning of the trial and after 8 weeks of training.

Although the studies described above are related to different physical disorders, they have major conclusions validating the findings of this paper. In general, they prove and emphasize the role of diaphragm in postural control. To our best knowledge, there is a lack of literature directly describing the role of diaphragm in the improvement of postural control after a subtalar joint injury.

Moreover, diaphragm may play such a strong postural role because of its fascial connections, as fascia is rich with Golgi's, Pacini's, and Ruffini's corpuscles [10]. Thoracolumbar fascia, fascia transversalis, and lateral raphe are all connected with diaphragm and provide proprioceptive information transmitted via diaphragm. Thus, every tissue surrounding fascia is characterized by different tensions, which is related with fascia restrictions and postural dysfunctions [10].

As it has already been explained in the introduction of the article, pressure differences created by the diaphragm influence and increase lymph flow [10]. Therefore, OMT of the diaphragm is substantiated to reduce swelling. A review article by Degenhardt and Kuchera [12], based on a number of scientific studies, also supported the use of OMT, directed at influencing the autonomic nervous system to improve lymphatic circulation. However, we are unaware of any previous study designed specifically to examine the diaphragm OMT efficacy in swelling reduction after a subtalar joint injury. We can only assume that the lack of an immediate effect in reducing oedema can be explained by the greater amount of time needed to increase lymphatic flow in the distal joints.

\section{Limitations}

The primary limitation of the study is the case study character. All the results are based on one subject only and therefore cannot be generalized to the population. There was no follow-up in the study so it is impossible to evaluate how long the postural effect of diaphragm release lasted and if reduction in swelling was achieved. However, the case has a strong base in anatomy and physiology. 


\section{Conclusion}

To the authors' knowledge, this is the first case report presented on OMT applied to the diaphragm that influenced a football player postural stability. The study confirmed the efficacy of the diaphragm OMT on improving postural control. At least one OMT session seems to be necessary to treat the diaphragm dysfunction with a substantial result. Screening for diaphragm dysfunction should be part of a complete osteopathic assessment in subtalar joint injuries. It is hoped that this case report will encourage research on diaphragm dysfunction in subtalar joint injuries.

\section{Disclosure statement}

No author has any financial interest or received any financial benefit from this research.

\section{Conflict of interest}

The authors state no conflict of interest.

\section{References}

1. Johnson U, Ekengren J, Andersen MB. Injury prevention in Sweden: helping soccer players at risk. J Sport Exerc Psychol. 2005;27(1):32-38; doi: 10.1123/jsep.27.1.32.

2. Paillard T, Noé F, Rivière T, Marion V, Montoya R, Dupui $P$. Postural performance and strategy in the unipedal stance of soccer players at different levels of competition. J Athl Train. 2006;41(2):172-176.

3. Wong $\mathrm{P}$, Hong $\mathrm{Y}$. Soccer injury in the lower extremities. Br J Sports Med. 2005;39(8):473-482; doi: 10.1136/ bjsm.2004.015511.

4. Yao S, Hassani J, Gagne M, George G, Gilliar W. Osteopathic manipulative treatment as a useful adjunctive tool for pneumonia. J Vis Exp. 2014;87:e50687; doi: 10.3791/50687.

5. Rocha T, Souza H, Brandão DC, Rattes C, Ribeiro L, Campos SL, et al. The Manual Diaphragm Release Technique improves diaphragmatic mobility, inspiratory capacity and exercise capacity in people with chronic obstructive pulmonary disease: a randomised trial. J Physiother. 2015;61(4):182-189; doi: 10.1016/j.jphys.2015.08.009.

6. Valenza MC, Cabrera-Martos I, Torres-Sánchez I, Garcés-García A, Mateos-Toset S, Valenza-Demet G. The effects of doming of the diaphragm in subjects with shorthamstring syndrome: a randomized controlled trial. J Sport Rehabil. 2015;24(4):342-348; doi: 10-1123/jsr.20140190.

7. Chikly BJ. Manual techniques addressing the lymphatic system: origins and development. J Am Osteopath Assoc. 2005;105(10):457-464.

8. Nason LK, Walker CM, McNeeley MF, Burivong W, Fligner CL, Godwin JD. Imaging of the diaphragm: anatomy and function. Radiographics. 2012;32(2):E51-E70; doi: 10.1148/rg.322115127.

9. Lane K, Worsley D, McKenzie D. Exercise and the lymphatic system: implications for breast-cancer survivors. Sports Med. 2005;35(6):461-471.

10. Bordoni B, Zanier E. Anatomic connections of the diaphragm: influence of respiration on the body system. $J$ Multidiscip Healthc. 2013;6:281-291; doi: 10.2147/JMDH. S45443.

11. Quick CM, Venugopal AM, Dongaonkar RM, Laine GA, Stewart RH. First-order approximation for the pressureflow relationship of spontaneously contracting lymphangions. Am J Physiol Heart Circ Physiol. 2008;294(5): H2144-H2149; doi: 10.1152/ajpheart.00781.2007.
12. Degenhardt BF, Kuchera ML. Update on osteopathic medical concepts and the lymphatic system. J Am Osteopath Assoc. 1996;96(2):97-100.

13. Kulkarni NV. Clinical anatomy (a problem solving approach), $2^{\text {nd }}$ edition. New Delhi: Jaypee Brothers Medical Publishers; 2011.

14. Souilamas R, Hidden G, Riquet M. Mediastinal lymphatic efferents from the diaphragm. Surg Radiol Anat. 2001; 23(3):159-162; doi: 10.1007/s00276-001-0159-y.

15. Kolar P, Sulc J, Kyncl M, Sanda J, Neuwirth J, Bokarius AV, et al. Stabilizing function of the diaphragm: dynamic MRI and synchronized spirometric assessment. J Appl Physiol. 2010;109(4):1064-1071; doi: 10.1152/japplphysiol.01216. 2009.

16. Hodges PW, Gandevia SC. Activation of the human diaphragm during a repetitive postural task. J Physiol. 2000; 522(1):165-175; doi: 10.1111/j.1469-7793.2000.t01-100165.xm.

17. Smith CE, Nyland J, Caudill P, Brosky J, Caborn DNM. Dynamic trunk stabilization: a conceptual back injury prevention program for volleyball athletes. J Orthop Sports Phys Ther. 2008;38(11):703-720; doi: 10.2519/jospt. 2008.2814.

18. Jung $\mathrm{H}$, Yamasaki M. Association of lower extremity range of motion and muscle strength with physical performance of community-dwelling older women. J Physiol Anthropol. 2016;35:30; doi: 10.1186/s40101-016-0120-8.

19. Licciardone JC, Kearns CM. Somatic dysfunction and its association with chronic low back pain, back-specific functioning, and general health: results from the OSTEOPATHIC trial. J Am Osteopath Assoc. 2012;112(7): 420-428.

20. Licciardone JC, Nelson KE, Glonek T, Sleszynski SL, Cruser A. Osteopathic manipulative treatment of somatic dysfunction among patients in the family practice clinic setting: a retrospective analysis. J Am Osteopath Assoc. 2005;105(12):537-544.

21. Mawdsley RH, Hoy DK, Erwin PM. Criterion-related validity of the figure-of-eight method of measuring ankle edema. J Orthop Sports Phys Ther. 2000;30(3):149-153; doi: 10.2519/jospt.2000.30.3.149.

22. Pugia ML, Middel CJ, Seward SW, Pollock JL, Hall RC, Lowe $\mathrm{L}$, et al. Comparison of acute swelling and function in subjects with lateral ankle injury. J Orthop Sports Phys Ther.2001;31(7):384-388;doi:10.2519/jospt.2001.31.7.384.

23. Hass CJ, Bishop MD, Doidge D, Wikstrom EA. Chronic ankle instability alters central organization of movement. Am J Sports Med. 2010;38(4):829-834; doi: 10.1177/03 63546509351562.

24. Hertel J. Sensorimotor deficits with ankle sprains and chronic ankle instability. Clin Sports Med. 2008;27(3): 353-370; doi: 10.1016/j.csm.2008.03.006.

25. Van Deun S, Staes FF, Stappaerts KH, Janssens L, Levin O, Peers KK. Relationship of chronic ankle instability to muscle activation patterns during the transition from double-leg to single-leg stance. Am J Sports Med. 2007;35(2):274-281; doi:10.1177/0363546506294470.

26. Terada M, Kosik KB, McCann RS, Gribble PA. Diaphragm contractility in individuals with chronic ankle instability. Med Sci Sports Exerc. 2016;48(10):2040-2045; doi: $10.1249 / M S S .0000000000000994$.

27. Vostatek P, Novák D, Rychnovský T, Rychnovská S. Diaphragm postural function analysis using magnetic resonance imaging. PLoS One. 2013;8(3):e56724; doi: 10.1371/journal.pone.0056724. 
28. Kolar P, Sulc J, Kyncl M, Sanda J, Cakrt O, Andel R, et al. Postural function of the diaphragm in persons with and without chronic low back pain. J Orthop Sports Phys Ther. 2012;42(4):352-362; doi: 10.2519/jospt.2012.3830.

29. Hodges PW, Heijnen I, Gandevia SC. Postural activity of the diaphragm is reduced in humans when respiratory demand increases. J Physiol. 2001;537(3):999-1008; doi: 10.1111/j.1469-7793.2001.00999.x.

30. Janssens L, McConnell AK, Pijnenburg M, Claeys K, Goossens N, Lysens R, et al. Inspiratory muscle training affects proprioceptive use and low back pain. Med Sci Sports Exerc. 2015;47(1):12-19; doi: 10.1249/MSS.00000000 00000385. 